\title{
Evaluation of Nested Polymerase Chain Reaction targeting hsp65 of Mycobacterium tuberculosis for the Detection of Organism in the Sputum Samples
}

\author{
${ }^{1}$ Yogaraje GC Varadaiah, ${ }^{2}$ Akila Prashant, ${ }^{3}$ Raghu K Chinnappa, ${ }^{4}$ Rakshitha M Nagaraj \\ ${ }^{5}$ Thejashwini, ${ }^{6}$ Robinson K Samuel, ${ }^{7}$ Devananda Devegowda, ${ }^{8}$ Prashant Vishwanath
}

\begin{abstract}
Introduction: The poor sensitivity of conventional smear microscopy and the delay in obtaining Mycobacterium culture results prevent the early diagnosis of Myobacterium tuberculosis (MTB). By using nucleic acid amplification techniques like polymerase chain reaction (PCR), one may be able to diagnose the disease on the day of arrival of specimen in the laboratory. The present study aimed to evaluate the applicability of the nested-PCR (nPCR) technique as a rapid and direct molecular method for the diagnosis of $M$. tuberculosis in sputum specimens of patients whose sputum smear was acid-fast bacilli (AFB) negative using heat shock protein ( $h s p 65)$ as the gene target.
\end{abstract}

Materials and methods: Early morning sputum samples were collected in sterile containers respectively from about 40 suspected patients of pulmonary tuberculosis, attending the outpatient units of JSS Medical College and PKTB Hospital, Mysore and from 20 age and sex-matched healthy controls. Sputum samples were decontaminated by modified Petroff's method and DNA was isolated using QIAGEN DNA extraction kit. The $\mathrm{nPCR}$ was carried out for the detection of MTB using the target gene $h s p 65$.

Results: Nested-PCR showed specific amplification (165bp) of M. tuberculosis in 18 out of 20 sputum AFB positive samples and 9 out of 20 AFB negative samples. None of the healthy controls showed any amplification with $\mathrm{nPCR}$. The $\mathrm{nPCR}$ when compared to that of Ziehl-Neelsen staining had a sensitivity of $90 \%$, specificity of $77.5 \%$, positive predictive value (PPV) of $66.6 \%$, and negative predictive value (NPV) of $93.9 \%$. The percentage of false positive was $33.3 \%$ and percentage of false negative was $6.1 \%$.

Conclusion: The detection of $M$. tuberculosis with nPCR in smear negative patients provides the bacteriological data 4 to 8 weeks earlier. A molecular approach, based on the amplification of hsp65 gene by nPCR, showed that there is high probability of the disease being absent when the test is negative because of the high negative predictive value (NPV).

Keywords: Heat shock protein (hsp65), Myobacterium tuberculosis, Nested-polymerase chain reaction, Ziehl-Neelsen staining.

\footnotetext{
${ }^{1-8}$ Department of Biochemistry, Center of Excellence in Molecular Biology and Regenerative Medicine, JSS Medical College and Hospital, JSS University, Mysore, Karnataka, India

Corresponding Author: Akila Prashant, Professor, Department of Biochemistry, Center of Excellence in Molecular Biology and Regenerative Medicine, JSS Medical College and Hospital, JSS University, Mysore, Karnataka, India, Phone: +919008097970 e-mail: akila.prashant@gmail.com
}

How to cite this article: Varadaiah YGC, Prashant A, Chinnappa RK, Nagaraj RM, Thejashwini, Samuel RK, Devegowda D, Vishwanath P. Evaluation of Nested Polymerase Chain Reaction targeting hsp65 of Mycobacterium tuberculosis for the Detection of Organism in the Sputum Samples. Indian J Med Biochem 2016;1(1):28-33.

Source of support: Nil

Conflict of interest: None

\section{INTRODUCTION}

Tuberculosis (TB) remains a global health problem. In 2012, an estimated 8.6 million people developed TB and 1.3 million died from the disease (including 320,000 deaths among HIV-positive people). ${ }^{1}$ The number of TB deaths is unacceptably large given that most are preventable. The increasing number (advent) of AIDS patients has made the disease a major public health and about $21 \%$ of the world's TB-infected population is in India., ${ }^{2,3}$ Although the pulmonary system is the most common location for $\mathrm{TB}$, extra pulmonary TB (EPTB) disease, resulting from spread of Myobacterium tuberculosis (MTB) from lungs to other tissues and organs through bloodstream, occurs in more than $20 \%$ of immunocompromised patients. In India and other developing countries, lymph node TB is among a major form of EPTB caused by nontuberculous mycobacteria species.

A rapid and an early diagnosis of $T B$ is thus an essential step to combat this disease. The conventional methods adopted for the diagnosis of TB include medical history, tuberculin skin test, chest $\mathrm{x}$-rays, bacteriological examination by the acid-fast bacilli (AFB) and mycobacterial culture. Sputum smear microscopy with Ziehl-Neelsen ( $\mathrm{ZN})$ staining is the most widely used rapid method in most laboratories for diagnosing TB under the Revised National Tuberculosis Control Program. The poor sensitivity of conventional smear microscopy and the delay in obtaining Mycobacterium culture results prevent the early diagnosis of TB. The difficulties of identifying mycobacteria in patient specimens emphasize the need for rapid and sensitive diagnostic methods for TB.

Recently, molecular tests based on nucleic acid amplification (NAA) technologies, such as polymerase 
chain reaction (PCR) have been shown to be a useful tool for the detection of infectious pathogens, such as MTB. The advantage of this method is that it can provide results within 1 day of collection of specimens, with a high degree of sensitivity and specificity. ${ }^{4}$ Also, PCR is able to detect less than 10 bacilli per $\mathrm{mL}$ from a variety of clinical specimens. 5,6 However, despite being promising, the sensitivity of these methods is sometimes low mainly in samples with negative AFB smear. ${ }^{7}$ For smear and culture negative specimens, PCR is the only currently available method that can provide bacteriological diagnosis. The development of PCR assays has used different genomic targets, such as IS6110, $16 S$ rDNA, rpoB, recA, and heat shock protein (hsp65), which have shown discrepant results in sensitivity.

The gene encoding the $65-\mathrm{kDa} h s p 65$ has been reported as an ideal target for $M$. tuberculosis detection by PCR as it is estimated to be highly conserved among mycobacterial species and present in all mycobacteria. It is more variable than the $16 S \mathrm{rRNA}$ gene sequence and for this reason it is a potential tool for the identification of genetically related species of mycobacteria.

Although conventional PCR is very useful for detection of $M$. tuberculosis, a variation of PCR, known as nestedPCR (nPCR) uses two sets of oligonucleotide primers in subsequent reactions, in which the amplification product of the first reaction is used as a template for the second reaction. This technique has been proposed for the detection of $M$. tuberculosis in cases requiring high specificity and sensitivity. ${ }^{8-11}$

The purpose of this study is to evaluate the applicability of the nested-PCR technique as a rapid and direct molecular method for the diagnosis of $M$. tuberculosis in sputum samples of patients using hsp65 as the gene target. The results of the nPCR were then compared with the results of the conventional method to evaluate the efficacy of nPCR for the diagnosis of MTB in clinical samples.

\section{MATERIALS AND METHODS}

\section{Source of the Data}

All adult patients who were clinically suspected of pulmonary TB as per World Health Organization guidelines attending JSS Hospital/PKTB Hospital, Mysore form the source of material. Early morning expectorated sputum samples were collected in wide mouthed disposable sterile containers from each suspected patient of pulmonary TB. Ethical committee approval was obtained before the commencement of the study. A well-informed and written consent was obtained from each patient.

\section{Type of Study}

A pilot study.

\section{Sample Size}

A total of 20 confirmed (sputum AFB positive by $\mathrm{ZN}$ staining) pulmonary TB patients and 20 suspected (sputum AFB negative by ZN staining) pulmonary TB patients, and 20 healthy nontubercular controls without any symptoms of MTB and negative ZN staining were included in the study.

\section{Inclusion Criteria}

- The patients were selected based on the presence of clinical symptoms leading to suspect TB, such as prolonged cough, fever, without apparent cause, weight loss, and night sweat.

- The diagnosis of TB was made on gold standard criteria employed by the health services in the diagnostic definition of $\mathrm{TB}$, such as the combination of epidemiological, clinical and laboratory data (chest $\mathrm{x}$-ray, tuberculin test, sputum smear), and patient history.

- Based on sputum AFB results, they were divided into two groups: Group 1: Confirmed pulmonary tuberculosis and Group 2: Suspected pulmonary tuberculosis.

\section{Exclusion Criteria}

- Patients who were already on anti-TB treatment, immunocompromised, or under corticosteroid-based medication were excluded from the study.

\section{Method of Sample Collection}

A total of 5 to $10 \mathrm{~mL}$ of early morning sputum samples were collected from 40 patients included in the study group during the period from November 2013 to October 2014. All the samples were smeared and screened with conventional microbiological test, such as Ziehl-Neelsen acid fast staining for recording smear positivity.

\section{Isolation of DNA from Sputum Samples}

Majority of sputum specimens submitted to the laboratory are contaminated by more rapidly growing normal flora. These overgrow before tubercle bacilli start to grow. Therefore, specimens must be submitted to harsh digestion and decontamination procedure that liquefies organic debris and eliminates unwanted normal flora. $^{12}$

All currently available digesting/decontaminating agents are to some extent toxic to tubercle bacilli. Therefore 
to ensure survival of maximum number of bacilli in specimen, decontamination procedure must be precisely followed. As a general rule, contamination rate of 2 to $3 \%$ is acceptable in laboratories that process fresh specimens. A laboratory which experiences no contamination is probably using a method that kills too many of tubercle bacilli. $^{12}$

\section{Modified Petroff's Method}

The sputum samples were digested and decontaminated by the aseptic addition of equal volume of sterile N-acetyl-L-cysteine- $2 \% \mathrm{NaOH}-1.5 \%$ sodium citrate solution in a $15 \mathrm{~mL}$ centrifuge tube. The cap was tightened and then incubated at room temperature for 30 minutes, centrifuged at 10,000 rpm for 25 minutes. The supernatant was discarded slowly into a container with $5 \%$ phenol solution. The pellet was washed twice with phosphate buffered saline pH7.6 or sterile distilled water. The supernatant was discarded and the pellet was resuspended in $0.5 \mathrm{~mL}$ of sterile distilled water and the mixture was transferred to a microcentrifuge tube and $40 \mu \mathrm{L}(20 \mathrm{mg} / \mathrm{mL})$ of proteinase $\mathrm{K}$ and $8 \mu \mathrm{L}$ RNase (100 mg/mL) was added, mixed by vortex followed by the addition of equal volume of lysis buffer and vortexed. The mixture was transferred to the QIAamp Mini spin column. Further, DNA was extracted by following the procedure mentioned in the DNA extraction kit (QIAGEN, Germany). The DNA sample eluted was stored in $-20^{\circ} \mathrm{C}$ until use.

\section{Measurement of DNA Concentration}

The spectrophotometric analysis and agarose gel electrophoresis was used to reveal the concentration and the purity of the genomic DNA. Elution buffer was used to dilute samples and to calibrate the spectrophotometer and measure the absorbance at 260, 280, and $320 \mathrm{~nm}$ using quartz microcuvette. The amount of ultraviolet (UV) radiation absorbed by a solution of DNA is directly proportional to the amount of DNA in the sample. For pure sample of DNA, the ratio of absorbance at 260 and $280 \mathrm{~nm}\left(\mathrm{~A}_{260} / \mathrm{A}_{280}\right)$ is between 1.6 and 1.9. $10 \mu \mathrm{L}$ of each DNA sample was loaded onto $1 \%$ agarose gel and the result was documented.

\section{Nested-PCR Amplification of hsp65 Gene and Detection of Mycobacterial DNA}

Following extraction, a nPCR for the detection of MTB was performed from DNA samples. Oligonucleotide primers (Eurofin) were synthesized and primary PCR was performed utilizing a pair of oligonucleotide primers, TB-1(5'-GAGATCGAGCTGGAGGATCC-3') and TB-2(5'-AGCTGCAGCCCAAAAGGTGTT-3'). For primary PCR, $5 \mu \mathrm{L}$ of stored DNA (150 ng) was used. The DNA was amplified in $50 \mu \mathrm{L}$ of reaction mixture, which was composed of $10 \times$ PCR buffer (10 mMTrisHCl [pH8.3], $50 \mathrm{~mm} \mathrm{KCL,} 2.5$ mm MgCl 2 ), $2.5 \mathrm{~mm}$ each of deoxynucleoside triphosphates (i.e., deoxyadenosine triphosphate, deoxycytosine triphosphate, deoxyguanosine triphosphate, and deoxythymidine triphosphate) (Himedia) and 50 picomol of $0.3 \mathrm{~mm}$ oligonucleotide primers, TB-1 and TB-2. Primary PCR was completed by adding $2.5 \mathrm{U}$ Taq DNA polymerase (Himedia) to this mixture. The samples were subjected to 40 amplification cycles consisting of 1.5 minutes denaturation at $95^{\circ} \mathrm{C}, 2$ minutes annealing at $60^{\circ} \mathrm{C}$, and 2 minutes extension at $72^{\circ} \mathrm{C}$ and with a final extension at $72^{\circ} \mathrm{C}$ for 10 minutes. The PCR reaction cycles were performed within an automated Thermal Cycler (Master Cycler Gradient, Eppendorff). For the nPCR of tuberculosis, the $10 \mu \mathrm{L}$ of primarily amplified DNA product was used as a template and the same procedure as primary PCR was performed, except for the utilization of a second pair of oligonucleotide primers. TB-3 (5'-CCATCGATCCGAGACCCTGCTCAAGGGC-3') and TB-4 (5'-TGCTCTAGACTCCTCGACGGTGATGACG-3'). DNA from the standard strain of MTB H37Rv was used as a positive control for the nPCR and the PCR solution without DNA template were used as negative controls.

\section{Analysis of the Amplified Products/Gel Electrophoresis of Amplified DNA}

An aliquot of $10 \mu \mathrm{L}$ nested or second PCR products were analyzed by gel electrophoresis in $2 \%$ agarose gel prepared in Tris-Acetate ethylenediaminetetraacetic acid buffer $\mathrm{pH} 8.6$ containing $0.5 \mu \mathrm{g} / \mathrm{mL}$ of ethidium bromide. The gel was run at 100 volts and then visualized under UV transilluminator. Further, the gel was examined in a gel documentation system (SynGene) for a $165 \mathrm{bp}$ product using standard molecular marker and the results were documented. Presence of a single band equivalent to $165 \mathrm{bp}$ was taken as a positive result when negative control gave no reaction. All the false negative and doubtful results were retested.

Throughout the PCR processing, recommended stringent precautions were followed and the results were evaluated in the light of performance of appropriate positive and negative controls, to avoid cross-contamination and false positive reactions. All the necessary clinical details were also taken. Known treated positive TB cases, failure cases, defaulter cases, and relapse cases were not considered for this study. 
Evaluation of Nested Polymerase Chain Reaction targeting hsp65 of Mycobacterium tuberculosis

\section{Determination of Sensitivity}

To assess the sensitivity of the nPCR assay, all the DNA samples were diluted to get concentration ranging from 1 to $50 \mathrm{ng}$ and each concentration was taken separately for testing by nPCR. It was observed that the nPCR was able to detect the MTB even at $30 \mathrm{ng}$ DNA concentration.

\section{Determination of Specificity}

Primers amplified successfully MTB DNA to give a $165 \mathrm{bp}$ product and there were no nonspecific amplified products in all the DNA samples tested. These results demonstrate the exquisite specificity of the amplified sequence of MTB.

\section{RESULTS}

The characteristics of the patients included in the study are shown in Table 1. Among the 20 AFB positive cases included in the study, 17 were males and 3 were females.
Table 1: Physical characteristics of the patients included in the study

\begin{tabular}{lll}
\hline & Mean & Standard deviation \\
\hline Age (years) & 42.70 & 15.647 \\
Body weight $(\mathrm{kg})$ & 57.10 & 9.907 \\
Height $(\mathrm{cm})$ & 160.98 & 7.238 \\
Body mass index & 22.047 & 3.6485 \\
Sex (M/F) & $48 / 12$ & \\
\hline
\end{tabular}

Among the 20 AFB negative patients included in the study 16 were males and 4 were females. Among the 20 healthy controls, 15 were males and 5 were females. The age of the patients recruited in the study ranged from 20 to 78 years. Among these, 10 were below 25, 34 between the age group 26 and 50, and 16 were above 50 years of age.

Nested-PCR showed specific amplification (165 bp) of MTB in 18 out of 20 sputum AFB positive samples (Fig. 1) and 9 out of 20 AFB negative samples (Fig. 2). In experiments for detection of lower limit of detection, the assay was able to detect the MTB even at $30 \mathrm{ng}$

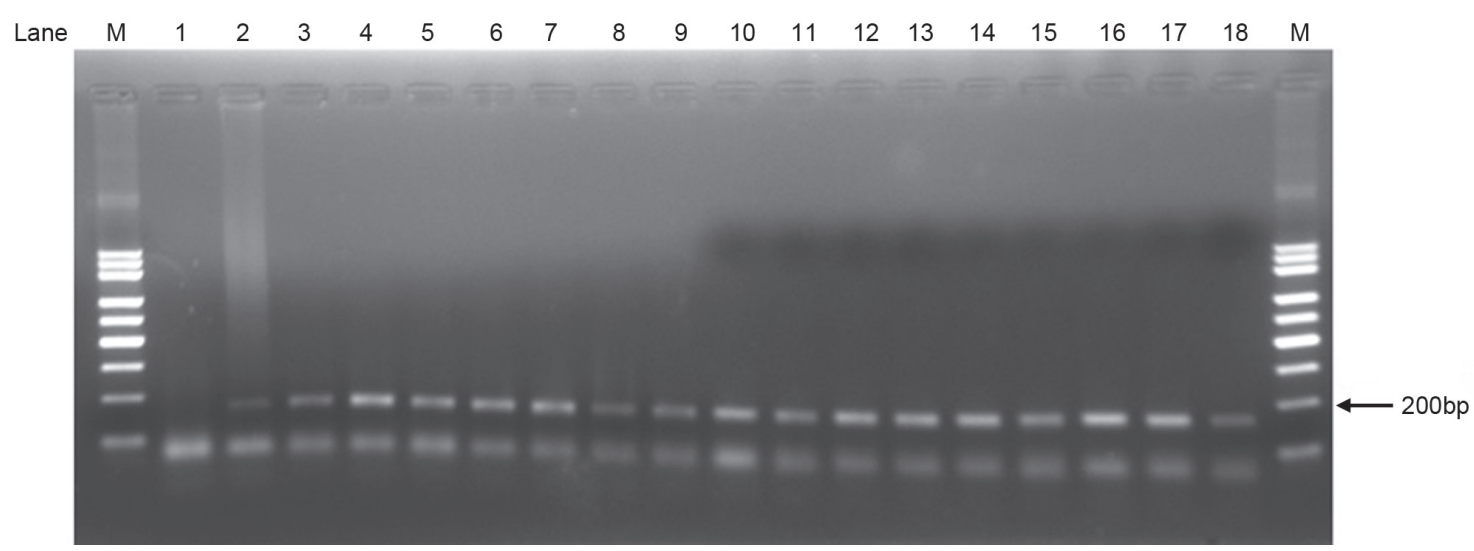

Fig. 1: Amplification products of PCR from MTB in sputum smear AFB positive samples. Polymerase chain reaction showing 165 bp target sequence amplified in case samples. Lane $1=$ Negative control, Lane $2=$ Positive control, Lane 3-18 = AFB positive cases, $M=100$ bp DNA ladder

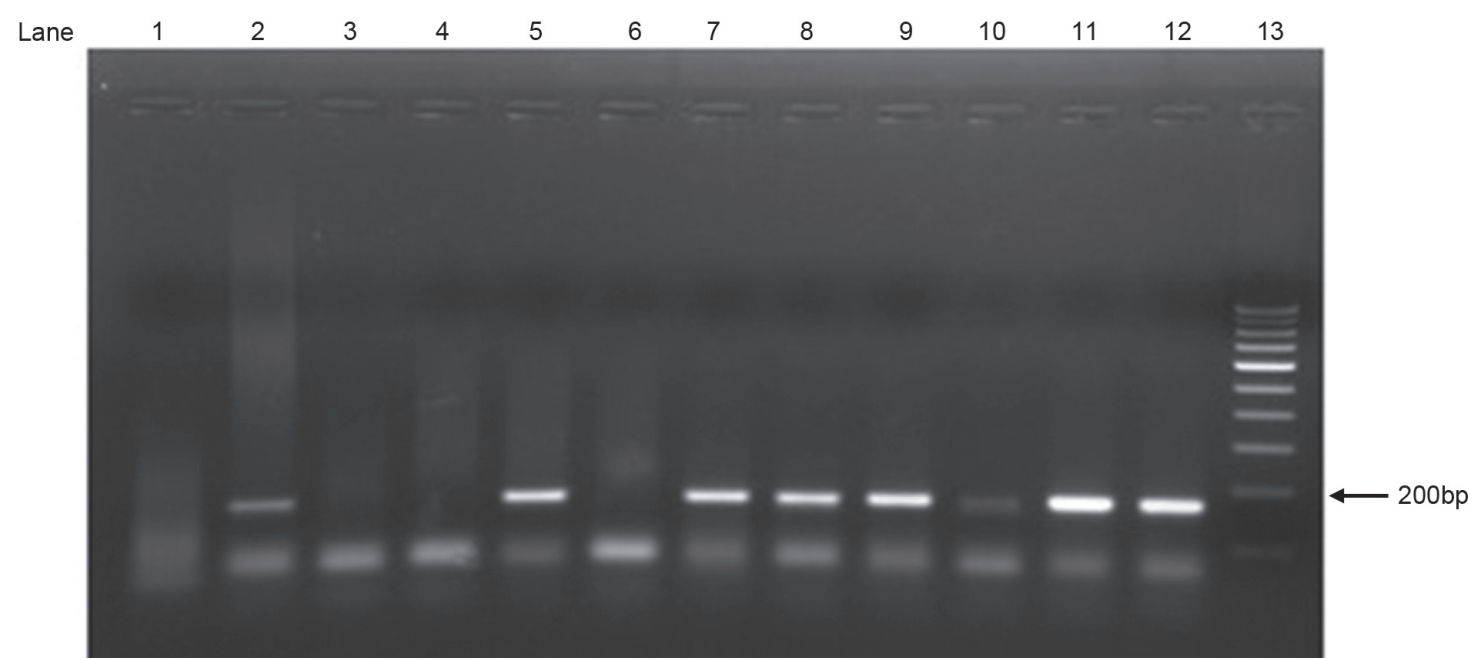

Fig. 2: Amplification products of PCR from MTB in sputum smear AFB negative samples. Polymerase chain reaction showing 165 bp target sequence amplified in case samples. Lane $1=$ Negative control, Lane $2=$ Positive control, Lane 3-12 = AFB negative cases, $M=100$ bp DNA ladder 
Table 2: Comparison of AFB staining and $\mathrm{nPCR}$

\begin{tabular}{llllll}
\hline & $\begin{array}{l}\text { AFB } \\
\text { positive } \\
(n=20)\end{array}$ & $\begin{array}{l}\text { AFB } \\
\text { negative } \\
n=40\end{array}$ & Total & $\begin{array}{l}\text { Chi- } \\
\text { square } \\
\text { test }\end{array}$ & $\begin{array}{l}p \text { value } \\
\text { val }\end{array}$ \\
\hline $\begin{array}{l}\text { nPCR } \\
\text { positive }\end{array}$ & $18(66.6 \%)$ & $9(33.3 \%)$ & $27(45 \%)$ & 7.025 & 0.020 \\
$\begin{array}{l}\text { nPCR } \\
\text { negative }\end{array}$ & $2(6.1 \%)$ & $31(93.9 \%)$ & $33(55 \%)$ & & \\
\hline Total & $20(33.3 \%)$ & $40(66.6 \%)$ & $60(100.0 \%)$ & \\
\hline
\end{tabular}

nPCR: Nested-polymerase chain reaction

DNA concentration. The results of the comparison of AFB staining and nPCR is shown in Table 2. Among the 20 healthy nontubercular controls included in the study, all the samples were negative for smear as well as nPCR. In this study, we have also evaluated the sensitivity, specificity, positive predictive value (PPV), negative predictive value (NPV) of the nPCR against the most widely used ZN smear conventional method. The nPCR when compared to that of $\mathrm{ZN}$ staining had a sensitivity of $90 \%$, specificity of $77.5 \%$, PPV of $66.6 \%$, and NPV of $93.9 \%$. The percentage of false positive was $33.3 \%$ and percentage of false negative was $6.1 \%$.

\section{DISCUSSION}

In India, most of the time the diagnosis of tuberculosis is primarily based on clinical features and gold standard methods, such as demonstration of AFB and culture of MTB. Since these gold standard methods fail to give results due to paucibacillary nature of the sample, NAA method to detect MTB in clinical specimens is increasingly used as a tool for TB diagnosis. Application of molecular methods in routine for diagnosing in developing country like ours depends on various factors like high cost and availability of skilled personnel to perform the test.

In the present study, 40 clinically suspected pulmonary TB cases admitted at JSS Medical College and PKTB Hospital, Mysore were studied along with 20 age and sex-matched healthy controls. DNA isolated from the sputum sample of 20 clinically suspected pulmonary TB whose sputum smear result was negative showed positive for TB in nine subjects by nPCR. However, the DNA isolated from 20 clinically suspected TB patients whose sputum smear result was positive for TB showed 18 positive by nPCR. The two subjects who showed a negative result by $\mathrm{nPCR}$ were reconfirmed and then reported. The negative result may be due to low copy number of bacilli or problem in digestion and decontamination of sputum sample. Also, the sputum is known to contain several inhibitors of PCR which makes successful amplification of mycobacterial DNA targets a challenge. ${ }^{13}$

It is widely accepted that $\mathrm{NPCR}$ is a new and rapid technique for the diagnosis of bacterial DNA with high specificity. ${ }^{8-11}$ By applying the $\mathrm{nPCR}$ technique, the RAPID BAP-MTB assay was able to detect as little as $10 \mathrm{fg}$, or the equivalent of 1 to 20 copies of MTB complex genomic DNA. ${ }^{14}$ Evaluation of nPCR targeting IS6110 of MTB using buffy coat of venous blood showed a sensitivity of $100 \%$ and a specificity of $95.1 \%$. The lower limit of detection in that study was less than 1 genome copy per microliter. ${ }^{15}$ The detection limit in whole blood using non-nested format was 20 cells per microliter. ${ }^{16}$ In our study, the lower limit of detection in terms of DNA concentration was $30 \mathrm{ng}$. We have used hsp65 as a target for the identification of MTB since several false positive results were reported questioning the specificity of IS6110 target. ${ }^{17,18}$ Previously reported sensitivity of $h s p 65 \mathrm{nPCR}$ with restriction analysis compared to microscopy and culture was 100\% (26/26 and $27 / 27$ respectively). Specificity and PPV were $93.1 \%$ (94/101) and $78.8 \%$ compared with microscopy and $95.0 \%(95 / 100)$ and $84.4 \%$ compared with culture $(P<0.05)$ respectively. ${ }^{19}$ Our study has shown a sensitivity of $90 \%$, specificity of $77.5 \%$, PPV of $66.6 \%$, and NPV of $93.9 \%$. Lower specificity shown in our study may be because the restriction analysis was not performed. Diagnosis of MTB using non-nested format of hsp 65 gene showed sensitivity of $81.13 \%$, with specificity of $88.24 \%$; the PPV and NPV were 95.56 and $60 \%$ respectively. ${ }^{20}$ However, the use of nested format showed that there is high probability of the disease being absent when the test is negative because of the high NPV.

Small sample size is the limitation of this study. Also, we have compared the nPCR results with $\mathrm{ZN}$ staining and the clinical criteria of the patients and not with culture. Using clinical criteria as gold standard is somewhat flawed due to the low specificity of symptoms of TB in relation to other diseases.

Hence, we would like to further evaluate this method in a large group of patients using other clinical specimens, such as blood, urine, pleural fluid, bronchial aspirate, lymph node aspirate, and compare the results with $\mathrm{ZN}$ staining, clinical criteria as well as with culture.

\section{ACKNOWLEDGMENTS}

Authors acknowledge the support received from VGST SPiCE, JSS University and DST FIST - 2012.

\section{REFERENCES}

1. "World Health organization: Global Tuberculosis control" WHO Report, 2013. Available from: http:/ / www.who.int/tb / publications/global report/en/index.html.

2. Hargreaves NJ, Kadzakumanja O, Phiri S, Nyangulu DS, Salaniponi FM, Harries AD, Squire SB. What causes smearnegative pulmonary tuberculosis in Malawi, an area of high 
HIV seroprevalence? Int J Tuberc Lung Dis 2001 Feb;5(2): 113-22.

3. Hudson CP, Wood R, Maartens G. Diagnosing HIV-associated tuberculosis: reducing costs and diagnostic delay. Int J Tuberc Lung Dis 2000 Mar;4(3):240-245.

4. AhmedN,MohantyAK,MukhopadhyayU, Batish VK, GroverS. PCR-based rapid detection of Mycobacterium tuberculosis in blood from immunocompetent patients with pulmonary tuberculosis. J Clin Microbiol 1998 Oct;36(10):3094-3095.

5. Honoré-Bouakline S, Vincensini JP, Giacuzzo V, Lagrange PH, Herrmann JL. Rapid diagnosis of extrapulmonary tuberculosis by PCR: impact of sample preparation and DNA extraction. J Clin Microbiol 2003 Jun;41(6):2323-2329.

6. Rodriguez JC, Fuentes E, Royo G. Comparison of two different PCR detection methods. Application to the diagnosis of pulmonary tuberculosis. APMIS 1997 Aug;105(8):612-616.

7. Lima SS, Clemente WT, Palaci M, Rosa RV, Antunes CM, Serufo JC. Conventional and molecular techniques in the diagnosis of pulmonary tuberculosis: a comparative study. J Bras Pneumol 2008 Dec;34(12):1056-1062.

8. Miyazaki Y, Koga H, Kohno S, Kaku M. Nested polymerase chain reaction for detection of Mycobacterium tuberculosis in clinical samples. J Clin Microbiol 1993 Aug;31(8):2228-2232.

9. Wu TL, Chia JH, Kuo AJ, Su LH, Wu TS, Lai HC. Rapid identification of mycobacteria from smear-positive sputum samples by nested PCR-restriction fragment length polymorphism analysis. J Clin Microbiol 2008;46(11):3591-3594.

10. Shukla I, Varshney S, Sarfraz, Malik A, Ahmad Z. Evaluation of nested PCR targeting IS6110 of Mycobacterium tuberculosis for the diagnosis of pulmonary and extrapulmonary tuberculosis. Biol Med 2011;3(2):171-175.

11. da Cruz HL, de Albuquerque Montenegro R, de Araújo Lima JF, da Rocha Poroca D, da Costa Lima JF, Maria Lapa Montenegro L, Crovella S, Charifker Schindler H. Evaluation of nested-PCR for mycobacterium tuberculosis detection in blood and urine samples. Braz J Microbiol 2011 Jan;42(1):321-329.

12. Singh A, Kashyap VK. Specific and rapid detection of mycobacterium tuberculosis complex in clinical samples by polymerase chain reaction. Interdiscip Perspect Infect Dis 2012;2012:654-694

13. Kesarwani RC, Pandey A, Misra A, Singh AK. Polymerase chain reaction $(\mathrm{PCR})$ : Its comparison with conventional techniques for diagnosis of extra-pulmonary tubercular diseases. Ind J Surg 2004;66:84-88.

14. Wang JY, Lee LN, Chou CS, Huang CY, Wang SK, Lai HC, Hsueh PR, Luh KT. Performance assessment of a nestedPCR assay (the RAPID BAP-MTB) and the BD ProbeTec ET system fordetection of Mycobacterium tuberculosis in clinical specimens. J Clin Microbiol 2004;42(10):4599-4603.

15. Nandagopal B, Sankar S, Lingesan K, Appu KC, Sridharan G, Gopinathan AK. Evaluation of nested PCR targeting IS6110 of Mycobacterium tuberculosis for detection of the organism in the leukocyte fraction of blood samples. Indian J Med Microbiol 2010 Jul-Sep;28(3):227-232.

16. Kox LF, Rhienthong D, Miranda AM, Udomsantisuk N, Ellis K, van Leeuwen J, van Heusden S, Kuijper S, Kolk AH. A more reliable PCR for detection of Mycobacterium tuberculosis in clinical samples. J Clin Microbiol 1994 Mar;32(3): 672-678.

17. Neonakis IK, Gitti Z, Krambovitis E, Spandidos DA. Molecular diagnostic tools in mycobacteriology. J Microbiol Methods 2008 Sep;75(1):1-11.

18. Sankar S, Kuppanan S, Balakrishnan B, Nandagopal B. Analysis of sequence diversity among IS6110 sequence of Mycobacterium tuberculosis: possible implicationsfor PCR based detection. Bioinformation 2011;6(7):283-285.

19. Macente S, Fujimura Leite CQ, Santos AC, Siqueira VL, Machado LN, Marcondes NR, Hirata MH, Hirata RD, Cardoso RF. Evaluation of hsp65 nested PCR-restriction analysis (PRA) for diagnosing tuberculosis in a high burden country. Biomed Res Int 2013;2013:391-549.

20. Zakham F, Bazoui H, Akrim M, Lemrabet S, Lahlou O, Elmzibri M, Benjouad A, Ennaji MM, Elaouad R. Evaluation of conventional molecular diagnosis of Mycobacterium tuberculosis in clinical specimens from Morocco. J Infect Dev Ctries 2012;6(1):40-45. 